\title{
Dopaminergic Terminals in the Nucleus Accumbens But Not the Dorsal Striatum Corelease Glutamate
}

\author{
Garret D. Stuber, ${ }^{1,2 *}$ Thomas S. Hnasko, ${ }^{3 *}$ Jonathan P. Britt, ${ }^{1,2}$ Robert H. Edwards, ${ }^{2,3}$ and Antonello Bonci ${ }^{1,2}$ \\ ${ }^{1}$ Ernest Gallo Clinic and Research Center, University of California, San Francisco, Emeryville, California 94608, and Departments of ${ }^{2} \mathrm{Neurology}$, \\ and ${ }^{3}$ Physiology, University of California, San Francisco, San Francisco, California 94143
}

Coincident signaling by dopamine and glutamate is thought to be crucial for a variety of motivated behaviors. Previous work has suggested that some midbrain dopamine neurons are themselves capable of glutamate corelease, but this phenomenon remains poorly understood. Here, we expressed the light-activated cation channel Channelrhodopsin-2 (ChR2) in genetically defined midbrain dopamine neurons to stimulate exocytosis specifically from dopaminergic terminals in both the nucleus accumbens (NAc) shell and dorsal striatum of brain slices from adult mice. Optical activation resulted in robust glutamate-mediated EPSCs in all medium spiny neurons examined in the NAc shell. In contrast, optically evoked glutamatergic currents were nearly undetectable in the dorsal striatum. Further, we used a conditional knock-out mouse lacking vesicular glutamate transporter 2 (VGLUT2) specifically in dopamine neurons to determine whether VGLUT2 is required for the exocytotic release of glutamate from dopamine neurons. Our data show that conditional knock-out completely abolished all optically evoked glutamate release. These results provide definitive physiological evidence for VGLUT2-mediated glutamate release by mature dopamine neurons projecting to the NAc shell, but not to the dorsal striatum. Thus, the unique ability of NAc-projecting dopamine neurons to synchronously activate both dopamine and glutamate receptors may have crucial implications for the ability to respond to motivationally significant stimuli.

\section{Introduction}

The role that a neuron plays within a circuit depends to a large extent on the neurotransmitter(s) released. Midbrain dopamine neurons project to a variety of forebrain targets, including the dorsal striatum and nucleus accumbens, where the release of dopamine is thought to contribute to the generation of motor function and motivated behaviors (Wise, 2004). As a variety of adaptive behaviors are ascribed to specific striatal subregions, it is possible that the striatal microcircuits that underlie these specific behavioral processes have distinct properties, such as differences in afferent or efferent connectivity.

Accumulating evidence suggests that a subset of midbrain dopamine neurons may also corelease the excitatory neurotransmitter glutamate both in vitro (Sulzer et al., 1998; Joyce and Rayport, 2000; Dal Bo et al., 2004) and in vivo (Chuhma et al., 2004; Lavin et al., 2005; Hnasko et al., 2010). However, this possibility has generated controversy due to difficulty stimulating selectively dopamine neurons or their terminals.

Vesicular glutamate transporters package glutamate into synaptic vesicles and are both necessary and sufficient for the exocy-

\footnotetext{
Received April 6, 2010; revised May 5, 2010; accepted May 11, 2010.

This work was supported by ABMRF and National Alliance for Research on Schizophrenia and Depression (G.D.S.), the A. P. Giannini Foundation (T.S.H.), and the State of California for Medical Research on Alcohol and Substance Abuse through the University of California, San Francisco (UCSF) (A.B.). We thank Kurt Thorn and the Nikon Imaging Center at UCSF for assistance and Karl Deisseroth for the AAV-DIO-ChR2-mcherry construct.

${ }^{*}$ G.D.S. and T.S.H. contributed equally to this work.

Correspondence should be addressed to Dr. Antonello Bonci, Department of Neurology and Ernest Gallo Clinic and Research Center, University of California, San Francisco, Emeryville, CA 94608. E-mail: antonello.bonci@ucsf.edu. DOI:10.1523/JNEUROSCI.1754-10.2010

Copyright $\odot 2010$ the authors $\quad 0270-6474 / 10 / 308229-05 \$ 15.00 / 0$
}

totic release of glutamate from neurons (Reimer and Edwards, 2004; Takamori, 2006). In culture, dopamine neurons immunostain for the vesicular glutamate transporter VGLUT2 (Dal Bo et al., 2004). However the histochemical evidence for VGLUT2 expression by dopamine neurons in vivo remains less clear. One study found substantial colocalization of mRNAs encoding the dopaminergic marker tyrosine hydroxylase (TH) and VGLUT2 in the ventral midbrain, with a higher incidence of colocalization in medial and caudal regions of the A10 dopamine cell group (Kawano et al., 2006). A second report detected colocalization only very rarely, but medial aspects were not examined (Yamaguchi et al., 2007). Using single-cell RT-PCR and immuno-electron microscopy, Trudeau and colleagues detected substantial colocalization of VGLUT2 transcript in dopamine neurons (Mendez et al., 2008) and VGLUT2 protein in TH+ terminals of the nucleus accumbens (Descarries et al., 2008; Bérubé-Carriére et al., 2009). However, the level of colocalization declined significantly across development (Mendez et al., 2008; Bérubé-Carrière et al., 2009), suggesting that glutamate corelease may similarly downregulate in adulthood.

To determine whether dopamine neurons release synaptically relevant amounts of glutamate in the adult brain, a method to selectively stimulate exocytotic release from dopamine terminals is required. We therefore used a multifaceted strategy, combining optogenetics and conditional gene disruption to selectively stimulate dopaminergic terminals while recording postsynaptic currents from medium spiny neurons (MSNs) in either the ventral or dorsal striatum of control or conditional knock-out mice lacking VGLUT2 specifically in dopamine neurons. 


\section{Materials and Methods}

Experimental subjects. Age-matched, sex-matched adult male and female mice ( $\sim 25 \mathrm{~g})$ were used as subjects. Control mice carried one copy of cre recombinase driven by dopamine transporter regulatory elements (Zhuang et al., 2005) and one conditional VGLUT2 allele (Hnasko et al., 2010) $\left(\right.$ Slc6a3 $^{+/ \text {cre }}$; Slc17a6 $\left.6^{+/ l o x}\right)$. Conditional VGLUT2 knock-out mice were identical but carried two conditional VGLUT2 alleles $\left(\mathrm{Slc}_{\mathrm{C} a 3^{+/ c r e}} ; \mathrm{Slc17a6^{lox/lox }}\right)$. Mice were group housed in a colony maintained with a standard $12 \mathrm{~h}$ light/dark cycle and given food and water ad libitum. Experiments were conducted in accordance with the Guide for the Care and Use of Laboratory Animals, as adopted by the National Institutes of Health, and with approval of the University of California, San Francisco Institutional Animal Care and Use Committee.

Stereotaxic recombinant adeno-associated $v i$ rus injection. Methods were adapted from (Tsai et al., 2009). Briefly, mice were anesthetized with ketamine/xylazine, placed in a stereotaxic frame (Kopf), the skull leveled, small holes drilled, and $1 \mu \mathrm{l}(0.25 \mu \mathrm{l} / \mathrm{min})$ of AAV5-EF1 $\alpha$ DIO-ChR2-mcherry $\left(\sim 3 \times 10^{12}\right.$ genomes $\left./ \mathrm{ml}\right)$ was injected using a Hamilton syringe bilaterally into the VTA (coordinates in mm relative to bregma: -3.25 anteroposterior, -4.50 dorsoventral, and \pm 0.5 mediolateral). Mice were allowed to recover for at least 3 weeks before further procedures.

Immunohistochemistry. Mice were perfused with cold PBS followed by $4 \%$ paraformaldehyde; their brains were removed, postfixed, cryoprotected in $30 \%$ sucrose, and frozen in superchilled isopentane; and $35 \mu \mathrm{m}$ sections were cut on a cryostat and floated in PBS. Sections were rinsed with PBS containing $0.2 \%$ Triton X-100, blocked $1 \mathrm{~h}$ with $4 \%$ normal donkey serum, and incubated overnight at $4^{\circ} \mathrm{C}$ in sheep anti-TH (Pel-Freze) and rabbit antiDsRed (Clontech), both at 1:2000 dilution in blocking solution. Sections were rinsed, incubated $2 \mathrm{~h}$ with Cy2conjugated anti-sheep and DyLite549-conjugated anti-rabbit secondaries (1:500, Jackson ImmunoResearch), rinsed again, mounted on slides, dehydrated through alcohol/xylenes, and coverslipped with DPX.

Images were collected using a Nikon Eclipse Ti-E motorized inverted microscope equipped with epifluorescence and a Photometrics Coolsnap HQ2 camera or a Nikon FN1 upright C1si spectral confocal microscope. For quantification of TH/ChR2-mcherry ${ }^{+}$neurons in the VTA, confocal images were taken using a $60 \times$ objective. $\mathrm{ChR} 2$-mcherry ${ }^{+}$neurons were identified and then scored for TH content.

Brain slice preparation. Mice were anesthetized and rapidly decapitated. Dissected brains were transferred to ice-cold artificial CSF (ACSF) containing the following (in $\mathrm{mM}$ ): 75 sucrose, $87 \mathrm{NaCl}, 2.5 \mathrm{KCl}, 7 \mathrm{MgCl}_{2}$, $0.5 \mathrm{CaCl}_{2}, 25 \mathrm{NaHCO}_{3}, 1.25 \mathrm{NaH}_{2} \mathrm{PO}_{4}$, and 1 ascorbic acid (saturated with $95 \% \mathrm{O}_{2}$ and $\left.5 \% \mathrm{CO}_{2}\right)$. Coronal sections of the striatum $(200 \mu \mathrm{m})$ were cut with a vibratome (VT1200, Leica). Slices were incubated for at least $30 \mathrm{~min}$ in a holding chamber containing the following (in $\mathrm{mM}$ ): 126 $\mathrm{NaCl}, 2.5 \mathrm{KCl}, 1.2 \mathrm{MgCl}_{2}, 2.5 \mathrm{CaCl}_{2}, 26 \mathrm{NaHCO}_{3}, 1.2 \mathrm{NaH}_{2} \mathrm{PO}_{4}, 11$ glucose, and 1 ascorbic acid (saturated with $95 \% \mathrm{O}_{2}$ and $5 \% \mathrm{CO}_{2}$ ). During recording, slices were superfused $(2 \mathrm{ml} / \mathrm{min})$ with this same ACSF at $\sim 32^{\circ} \mathrm{C}$ but with picrotoxin $\left(100 \mu \mathrm{M}\right.$, to block $\mathrm{GABA}_{\mathrm{A}}$ receptor-mediated synaptic currents) and without ascorbic acid.

Patch-clamp electrophysiology. Whole-cell voltage-clamp recordings from MSNs located in the nucleus accumbens (NAc) shell and dorsal

C.
B
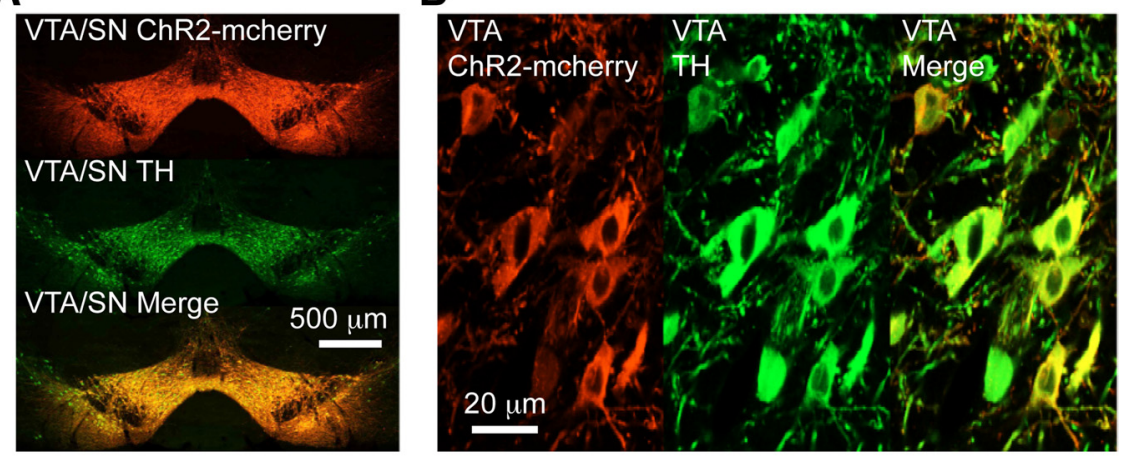

,

Figure 1. Expression of $C h R 2$-mcherry in midbrain dopamine neurons and their projections to the striatum. $\boldsymbol{A}$, Immunostaining for ChR2-mcherry (red) and TH (green) in the VTA/SN 30 d after injection of AAV-DI0-ChR2-mcherry in a mouse expressing cre neurons in the VTA colabel for TH. C, Staining as described above in a coronal plane including both DS and NAc shell. D, Confocal images of dopaminergic fibers in the NAc shell indicate that essentially all ChR2-mcherry-labeled puncta also contain TH.

striatum (DS) were obtained under visual control on a differential interference contrast, upright microscope with infrared illumination. Recordings were obtained using 3-6 M $\Omega$ resistance pipettes backfilled with internal solution containing the following (in mM): $120 \mathrm{CsCH}_{3} \mathrm{SO}_{3}, 20$ HEPES, 0.4 EGTA, $2.8 \mathrm{NaCl}, 5 \mathrm{~N}\left(\mathrm{CH}_{2} \mathrm{CH}_{3}\right)_{4} \mathrm{Cl}, 2.5 \mathrm{Mg}$-ATP, and 0.25 Mg-GTP, pH 7.3. Currents were measured using either an Axopatch 1D or $200 \mathrm{~A}$ amplifier (2 kHz low-pass Bessel filter) with a DigiData 1440 interface ( $5 \mathrm{kHz}$ digitization) and pClamp software (Molecular Devices). MSNs, identified by their morphology and hyperpolarized resting membrane potential, were voltage clamped at $-70 \mathrm{mV}$. An optical fiber $(200$ $\mu \mathrm{m}$ core diameter, 0.2 numerical aperture) coupled to a diode-pumped solid-state $473 \mathrm{~nm}$ laser was placed $200 \mu \mathrm{m}$ from the site of recording, and was used to deliver optical stimulation at a frequency of $0.1 \mathrm{~Hz}$. Stimulus intensity ranged from 1 to $30 \mathrm{~mW}$ with a pulse duration of $5 \mathrm{~ms}$. Series resistance (5-20 M 2 ) was monitored online with a $5 \mathrm{mV}$ hyperpolarizing step (50 ms) given after each stimulus.

To quantify EPSC amplitudes, six sweeps were collected from each cell at each light intensity $(0,1,2,5$, and $10 \mathrm{~mW}$ for input/output experiment, $30 \mathrm{~mW}$ for maximal stimulation and pharmacology experiments). Sweeps were then averaged offline to determine EPSC amplitude at a particular intensity. Light stimulus intensities were presented in a random order and counterbalanced across recordings. For AMPA receptor (AMPAR) antagonism experiments, $10 \mu \mathrm{M}$ 6,7-dinitroquinoxaline-2,3dione (DNQX) was used, and for dopamine (DA) receptor antagonism experiments, $2 \mu \mathrm{M} R$-(+)-7-chloro-8-hydroxy-3-methyl-1-phenyl-2,3,4,5- 
A

Nucleus Accumbens Shell C
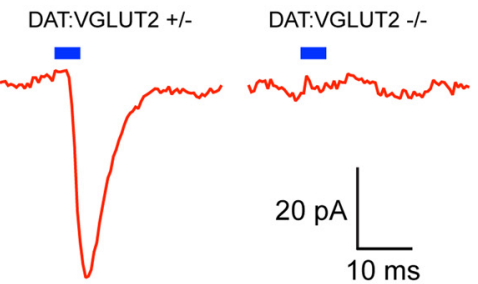

B
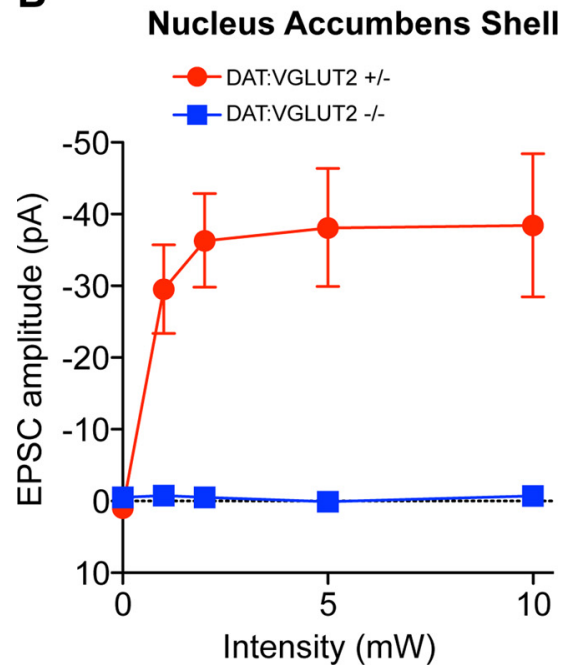

D

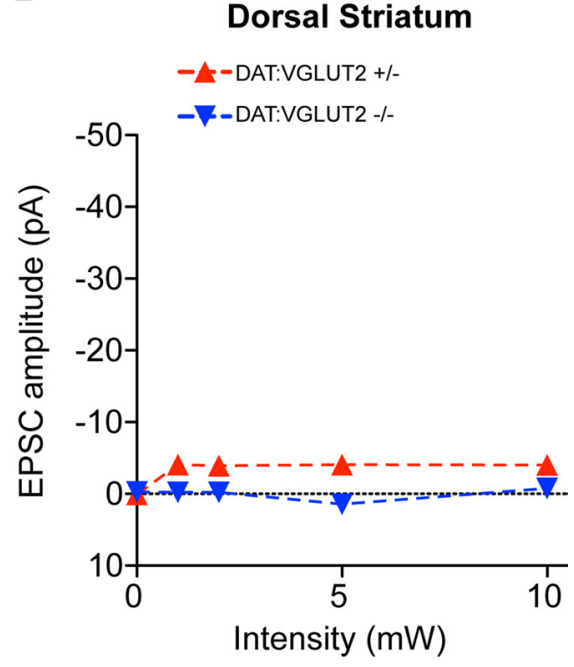

Figure 2. Dopaminergic terminals in the nucleus accumbens shell but not dorsal striatum corelease glutamate. $A$, Example EPSCs recorded in the NAc shell at $-70 \mathrm{mV}$ following a 5 ms blue light pulse at maximal intensity. $\boldsymbol{B}$, Light-evoked EPSC amplitudes recorded in the NAc shell were significantly greater in DAT:VGLUT2 heterozygous mice than in conditional knock-outs $(n=9-24$ cells per group; 2-way ANOVA for the interaction of genotype and light intensity: $\left.F_{(4,128)}=2.56, p<0.05\right)$. C, Example EPSCs recorded in the DS at $-70 \mathrm{mV}$ following a $5 \mathrm{~ms}$ blue light pulse at maximal intensity. $\boldsymbol{D}$, Light-evoked EPSC amplitudes recorded in the DS were not significantly different in DAT:VGLUT2 heterozygous mice versus conditional knock-outs as a function of light intensity (2-way ANOVA for the interaction of genotype and light intensity: $F_{(4,87)}=0.31, p=0.91$ ). EPSC amplitudes (independent of light intensity) were significantly different across genotypes $\left(F_{(1,87)}=12.80, p=0.006\right)$.

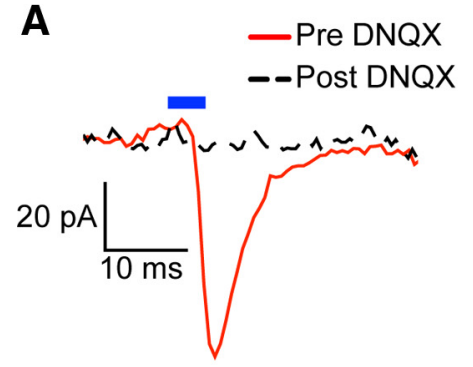

B

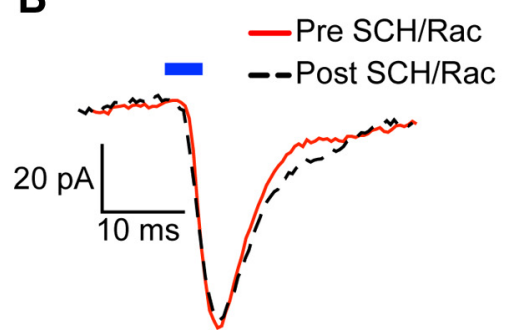

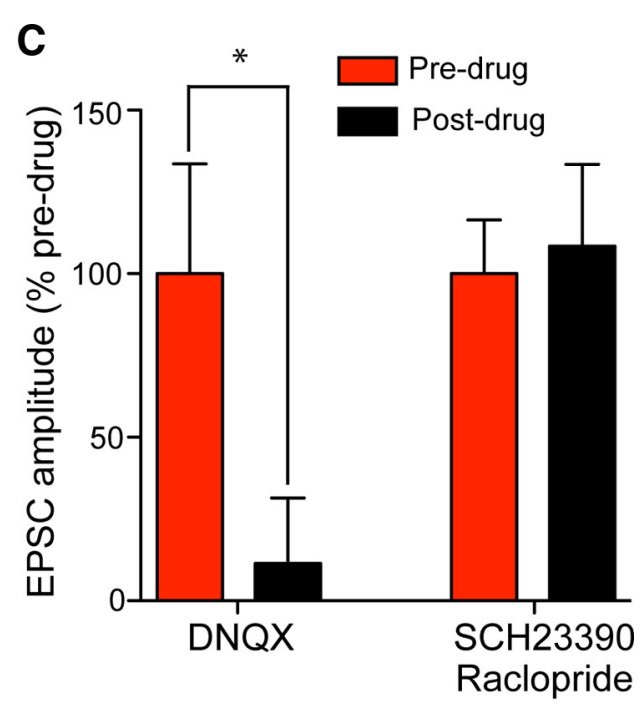

Figure 3. EPSCs evoked by dopamine terminal stimulation in the nucleus accumbens shell are blocked by AMPAR antagonism but unaffected by $D_{1} R / D_{2} R$ antagonism. $A$, Example EPSCs recorded at $-70 \mathrm{mV}$ in the NAc shell from DAT:VGLUT2 heterozygous mice before and after bath application of DNQX. $\boldsymbol{B}$, Example EPSCs recorded at $-70 \mathrm{mV}$ in the NAc shell from DAT:VGLUT2 heterozygous mice before and after bath application of SCH23390/raclopride. C, Light-evoked EPSCs from dopamine terminals were significantly reduced by DNQX bath application $\left(n=5\right.$ cells; $\left.t_{(4)}=2.61 ; p<0.05\right)$ but not by SCH23390/raclopride $(n=9$ cells; $t_{(8)}=0.40 ; p=0.70$ ). All data indicate mean \pm SEM. tetrahydro-1 $H$-3-benzazepine (SCH23390) and $2 \mu \mathrm{M}$ raclopride were used to block $\mathrm{D}_{1}$ and $\mathrm{D}_{2}$ receptors, respectively. Experimenters were blind to the animal's genotype during data acquisition.

Fast-scan cyclic voltammetry. Methods were adapted from previous work (Stuber et al., 2008). T-650 carbon fiber microelectrodes (100-200 $\mu \mathrm{m}$ in length) were used for detection of dopamine. Electrodes were placed in the NAc shell or dorsal striatum of Slc $6 a 3^{+/ c r e}$; Slc17a $6^{+/ l o x}$ mice. The potential applied to the electrode was ramped from $-0.4 \mathrm{~V}$ to $+1.3 \mathrm{~V}$ to $-0.4 \mathrm{~V}$ versus a $\mathrm{Ag} / \mathrm{AgCl}$ reference every 100 $\mathrm{ms}$ at a rate of $400 \mathrm{~V} / \mathrm{s}$, and resulting electrochemical data were acquired using customwritten software in LabVIEW. Electrochemical data were low-pass filtered at $1 \mathrm{kHz}$ offline. Background-subtracted cyclic voltammograms were generated immediately after optical stimulation of the slice, and were characteristic of dopamine (peak oxidation potential 600-700 mV). Current resulting from the oxidation of dopamine (monitored at the peak oxidation potential determined from cyclic voltammograms) were then converted into concentration changes using a calibration factor of $10 \mathrm{nA} / \mu \mathrm{M}$ DA.

\section{Results}

An adeno-associated virus encoding a conditional allele of the light-activated cation channel channelrhodopsin-2 (ChR2) activated by cre-mediated recombination (AAV5-DIO-ChR2-mcherry) (Tsai et al., 2009) was bilaterally injected into the ventral tegmental area (VTA) of adult mice (2-4 months old) expressing cre recombinase under the control of dopamine transporter (DAT) regulatory sequences (Zhuang et al., 2005). Four weeks after injection, immunohistochemistry revealed robust, selective expression of ChR2-mcherry in VTA dopamine neurons (Fig. $1 A, B$ ). Indeed, $99.5 \%$ of ChR2-mcherry ${ }^{+}$neurons identified in the VTA ( $n=409$ cells) were also labeled for the catecholamine biosynthetic enzyme TH. Furthermore, essentially all ChR2-mcherry-labeled fibers and puncta in striatal terminals costained for TH (Fig. 1C,D).

We then prepared coronal sections through striatal regions from injected mice and light-evoked stimulation was used to depolarize and stimulate neurotransmitter release from the dopaminergic terminals selectively expressing ChR2. Whole-cell voltage-clamp recordings from visually identified MSNs revealed that optical stimulation of dopaminergic terminals in the NAc shell resulted in EPSCs $>10$ pA in $24 / 24$ neurons, which increased in amplitude as a function of light stimulus intensity (Fig. 2A,B). Lightevoked EPSCs in the NAc shell were blocked by AMPAR antagonism (Fig. $3 A, C)$. The rapid response to light sug- 
gests a monosynaptic event, but to ensure that the glutamate-mediated currents did not reflect the postsynaptic action of dopamine, we applied $\mathrm{D}_{1}$ - and $\mathrm{D}_{2}$-type dopamine receptor antagonists to the bath. The addition of SCH23390 and raclopride did not significantly affect light-evoked EPSCs (Fig. 3 B, C), indicating that they do not require the light-evoked release of dopamine. Rather, dopamine neurons themselves appear to release glutamate.

The ability of dopamine neurons to release glutamate implies that they express a vesicular glutamate transporter. To determine whether VGLUT2 expression by dopamine neurons is required for their release of glutamate, we performed the same experiments in conditional knockout (cKO) mice (Slc6a3 ${ }^{+/ \text {cre }} ;$ Slc17a $6^{\text {lox/lox }}$ ) where cre recombinase drives both selective excision in dopamine neurons of the gene encoding VGLUT2 (Hnasko et al., 2010) and, following virus injection, the selective expression of ChR2 in dopamine neurons. In contrast to control mice, light never evoked EPSCs in cKO mice (Fig. $2 A, B)$, demonstrating that VGLUT2 is required for glutamate packaging and release from dopaminergic terminals.

Although previous results have suggested that mesolimbic dopamine neurons release glutamate (Chuhma et al., 2004; Hnasko et al., 2010), it is unknown whether dopamine neurons that project to the DS also have the capacity to release glutamate. Therefore, we also examined brain slices through the DS. Optical stimulation of dopaminergic terminals in the DS resulted in $\sim 10$-fold smaller mean glutamate-mediated currents than in the NAc shell of control mice, with no detectable release in the DS from mice lacking VGLUT2 in dopamine neurons (Fig. 2C,D). Importantly, light-evoked dopamine release as measured by fast-scan cyclic voltammetry was detected in both the DS (Fig. $4 A, C$ ) and NAc shell (Fig. $4 B, D$ ) of virally injected mice. Within the striatum, glutamate corelease is thus relatively restricted to dopamine terminals in the ventral striatum.

\section{Discussion}

Our results provide direct physiological evidence that mature dopamine neurons projecting to the NAc shell corelease glutamate. Previous studies have suggested that mesolimbic dopamine neurons release glutamate (Chuhma et al., 2004, 2009; Lavin et al., 2005; Hnasko et al., 2010), but electrical stimulation nonspecifically activates all VTA neurons, making it difficult to exclude glutamate release from nondopaminergic neurons. Indeed, there exist VGLUT2-positive neurons in the VTA that do not colabel for TH (Kawano et al., 2006; Yamaguchi et al., 2007; NairRoberts et al., 2008). Although some of these neurons appear to project locally (Dobi et al., 2010), it is unknown whether they may also project to striatal and/or limbic brain regions. Our ability to drive ChR2 expression selectively in dopamine neurons of the VTA and thus to selectively activate dopamine terminals in striatal regions with light eliminates the possibility of inadver-
B Nucleus Accumbens Shell

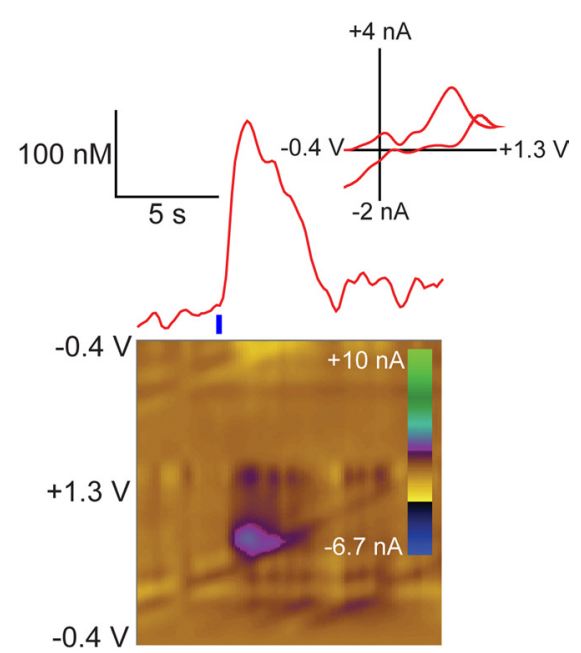

Nucleus Accumbens Shell

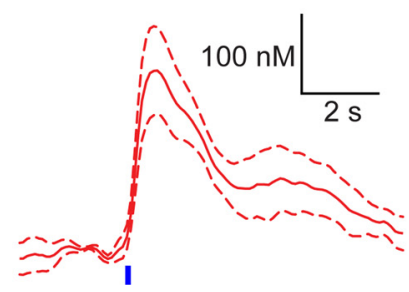

Figure 4. Optical stimulation of dopamine terminals in dorsal striatum and nucleus accumbens shell results in dopamine release. $A$, Example trace of dopamine release resulting from optical stimulation of dopamine terminals in the DS (1 pulse, $5 \mathrm{~ms}$ pulse duration). Inset shows background-subtracted cyclic voltammograms taken immediately after optical stimulation. The color plow shows current measured as the electrode across the entire scanned potential range. $\boldsymbol{B}$, Example trace of dopamine release resulting from optical stimulation of dopamine terminals in the NAc shell. C, Average dopamine release (solid line; dashed lines indicate $\pm S E M$ ) in the dorsal striatum following one-pulse optical stimulation ( $n=7$ recording sites). $\boldsymbol{D}$, Average dopamine release in the nucleus accumbens shell following one-pulse optical stimulation ( $n=5$ recording sites).

tently activating these nondopaminergic VTA glutamate neurons. Essentially all ChR2-mcherry-expressing neurons in the midbrain and puncta in the striatum were also labeled for $\mathrm{TH}$ by confocal microscopy (Fig. $1 B, D$ ). Additionally, no ChR2mcherry-expressing neurons were seen in brain regions known to send glutamatergic projections to the NAc shell, such as cortex, thalamus, hippocampus, amygdala, or lateral hypothalamus. Surprisingly, we did observe small clusters of ChR2-expressing neurons in both the lateral habenula and in the ventral premammillary nucleus (data not shown), but these neurons are not known to project to the NAc shell.

Due to technical limitations, earlier electrophysiological studies of glutamate corelease in the NAc shell have reported relatively small AMPAR currents limited to brain slices made from young ( $\sim 3$ weeks old) animals (Chuhma et al., 2004; Hnasko et al., 2010). Given that VGLUT2 expression by dopamine neurons appears to upregulate in culture (Dal Bo et al., 2004; Mendez et al., 2008) and downregulate over development in vivo (Mendez et al., 2008; Bérubé-Carrière et al., 2009), it has remained unclear whether mature dopamine neurons also corelease glutamate. However, we now find that VGLUT2 in dopamine neurons is required for glutamate release from dopaminergic terminals in the NAc shell of adult mice, demonstrating that VGLUT2dependent glutamate corelease persists into adulthood. Although some evidence suggests that only a small fraction of dopamine 
neurons express VGLUT2 in adult rodents (Yamaguchi et al., 2007), we see ubiquitous light-evoked glutamatergic currents in MSNs of the NAc shell. Therefore, it is likely that a significant number of dopamine neurons in the adult VTA express VGLUT2 at levels too low to detect by conventional means, or alternatively, a small percentage of VGLUT2-expressing dopamine neurons may send axon collaterals to essentially all MSNs in the NAc shell.

Although we were able to observe substantial ChR2-mcherry expression and light-evoked dopamine release in the DS, glutamate currents were much smaller and less frequent in the DS relative to the NAc shell. These results are consistent with a previous report (Kawano et al., 2006) showing the absence of VGLUT2 in the substantia nigra pars compacta compared to the VTA. More recently, we reported that cKO mice lacking VGLUT2 selectively in dopamine neurons show a presynaptic reduction in dopamine storage and release that is restricted to the ventral striatum (Hnasko et al., 2010). Together, these findings strongly suggest that within the striatum, VGLUT2 expression and glutamate corelease is restricted to mesolimbic projections, which may also extend to other limbic structures (Lavin et al., 2005).

In conclusion, our findings provide unequivocal evidence that a subset of dopaminergic terminals release glutamate capable of postsynaptic signaling. Mice lacking VGLUT2 in dopamine neurons show deficits in psychostimulant-induced locomotion (Hnasko et al., 2010; Wallén-Mackenzie et al., 2010), apparently due to a presynaptic role for glutamate in vesicular monoamine filling (Amilhon et al., 2010; Hnasko et al., 2010). Although we still understand little about the role for glutamate released by monoamine neurons as an independent signal, rapid, synaptic signaling mediated by glutamate may provide the temporal specificity required for reward prediction and/or incentive salience (Lapish et al., 2006, 2007).

\section{References}

Amilhon B, Lepicard E, Renoir T, Mongeau R, Popa D, Poirel O, Miot S, Gras C, Gardier AM, Gallego J, Hamon M, Lanfumey L, Gasnier B, Giros B, El Mestikawy S (2010) VGLUT3 (vesicular glutamate transporter type 3) contribution to the regulation of serotonergic transmission and anxiety. J Neurosci 30:2198-2210.

Bérubé-Carrière N, Riad M, Dal Bo G, Lévesque D, Trudeau LE, Descarries L (2009) The dual dopamine-glutamate phenotype of growing mesencephalic neurons regresses in mature rat brain. J Comp Neurol 517: $873-891$.

Chuhma N, Zhang H, Masson J, Zhuang X, Sulzer D, Hen R, Rayport S (2004) Dopamine neurons mediate a fast excitatory signal via their glutamatergic synapses. J Neurosci 24:972-981.

Chuhma N, Choi WY, Mingote S, Rayport S (2009) Dopamine neuron glutamate cotransmission: frequency-dependent modulation in the mesoventromedial projection. Neuroscience 164:1068-1083.

Dal Bo G, St-Gelais F, Danik M, Williams S, Cotton M, Trudeau LE (2004) Dopamine neurons in culture express VGLUT2 explaining their capacity to release glutamate at synapses in addition to dopamine. J Neurochem 88:1398-1405.
Descarries L, Bérubé-Carrière N, Riad M, Bo GD, Mendez JA, Trudeau LE (2008) Glutamate in dopamine neurons: synaptic versus diffuse transmission. Brain Res Rev 58:290-302.

Dobi A, Margolis EB, Wang HL, Harvey BK, Morales M (2010) Glutamatergic and nonglutamatergic neurons of the ventral tegmental area establish local synaptic contacts with dopaminergic and nondopaminergic neurons. J Neurosci 30:218-229.

Hnasko TS, Chuhma N, Zhang H, Goh GY, Sulzer D, Palmiter RD, Rayport S, Edwards RH (2010) Vesicular glutamate transport promotes dopamine storage and glutamate corelease in vivo. Neuron 65:643-656.

Joyce MP, Rayport S (2000) Mesoaccumbens dopamine neuron synapses reconstructed in vitro are glutamatergic. Neuroscience 99:445-456.

Kawano M, Kawasaki A, Sakata-Haga H, Fukui Y, Kawano H, Nogami H, Hisano S (2006) Particular subpopulations of midbrain and hypothalamic dopamine neurons express vesicular glutamate transporter 2 in the rat brain. J Comp Neurol 498:581-592.

Lapish CC, Seamans JK, Chandler LJ (2006) Glutamate-dopamine cotransmission and reward processing in addiction. Alcohol Clin Exp Res 30:1451-1465.

Lapish CC, Kroener S, Durstewitz D, Lavin A, Seamans JK (2007) The ability of the mesocortical dopamine system to operate in distinct temporal modes. Psychopharmacology (Berl) 191:609-625.

Lavin A, Nogueira L, Lapish CC, Wightman RM, Phillips PE, Seamans JK (2005) Mesocortical dopamine neurons operate in distinct temporal domains using multimodal signaling. J Neurosci 25:5013-5023.

Mendez JA, Bourque MJ, Dal Bo G, Bourdeau ML, Danik M, Williams S, Lacaille JC, Trudeau LE (2008) Developmental and target-dependent regulation of vesicular glutamate transporter expression by dopamine neurons. J Neurosci 28:6309-6318.

Nair-Roberts RG, Chatelain-Badie SD, Benson E, White-Cooper H, Bolam JP, Ungless MA (2008) Stereological estimates of dopaminergic, GABAergic and glutamatergic neurons in the ventral tegmental area, substantia nigra and retrorubral field in the rat. Neuroscience 152:10241031.

Reimer RJ, Edwards RH (2004) Organic anion transport is the primary function of the SLC17/type I phosphate transporter family. Pflugers Arch 447:629-635.

Stuber GD, Klanker M, de Ridder B, Bowers MS, Joosten RN, Feenstra MG, Bonci A (2008) Reward-predictive cues enhance excitatory synaptic strength onto midbrain dopamine neurons. Science 321:1690-1692.

Sulzer D, Joyce MP, Lin L, Geldwert D, Haber SN, Hattori T, Rayport S (1998) Dopamine neurons make glutamatergic synapses in vitro. J Neurosci 18:4588-4602.

Takamori S (2006) VGLUTs: 'exciting' times for glutamatergic research? Neurosci Res 55:343-351.

Tsai HC, Zhang F, Adamantidis A, Stuber GD, Bonci A, de Lecea L, Deisseroth $\mathrm{K}$ (2009) Phasic firing in dopaminergic neurons is sufficient for behavioral conditioning. Science 324:1080-1084.

Wallén-Mackenzie A, Wootz H, Englund H (2010) Genetic inactivation of the vesicular glutamate transporter 2 (VGLUT2) in the mouse: what have we learnt about functional glutamatergic neurotransmission? Ups J Med Sci 115:11-20.

Wise RA (2004) Dopamine, learning and motivation. Nat Rev Neurosci 5:483-494.

Yamaguchi T, Sheen W, Morales M (2007) Glutamatergic neurons are present in the rat ventral tegmental area. Eur J Neurosci 25:106-118.

Zhuang X, Masson J, Gingrich JA, Rayport S, Hen R (2005) Targeted gene expression in dopamine and serotonin neurons of the mouse brain. J Neurosci Methods 143:27-32. 